\title{
¿Leonardo da Vinci científico?
}

\author{
Leonardo da Vinci, scientist?
}

\author{
Maíra Pouey Bragos \\ Estudiante de Licenciatura en Filosofía, Escuela de Filosofía de la Facultad de Humanidades y Artes, \\ Universidad Nacional de Rosario - UNR, Rosario, Santa Fe, Argentina.
}

Resumén: El presente trabajo tiene como primer objetivo analizar la figura multifacética de Leonardo según dos autores: Giorgio Vasari y Karl Jaspers. A partir de la lectura de ambos autores, se expone cuáles son los aspectos que se tienen en cuenta a la hora de reconstruir una imagen del vinciano y en qué campo es ubicado por estos autores. El primero enfatiza que la característica primordial es ser un artista, mientras que el segundo propone que puede ser considerado además de un artista, un filósofo, mas de ninguna manera un científico. Creemos que el veredicto de Jaspers puede ser cuestionado. Aquí se introduce el segundo objetivo, discutir con Jaspers y establecer a partir de qué noción de ciencia se lo puede considerar a nuestro renacentista dentro de esta esfera. Para elucidar este punto se tendrá en cuenta la lectura de dos obras de Thomas Kuhn. Quizás adoptando una perspectiva kuhniana difiera el análisis que ha hecho la tradición de Leonardo en torno a su lugar dentro de la historia de la ciencia.

Palabras clave: epistemología, Leonardo da Vinci, historia de la ciencia.

Abstract: The aim of this paper is to analyze the multifaceted personality of Leonardo da Vinci. To do this, we will take into account the views of Giorgio Vasari and Karl Jaspers. From the study of both authors, the main features defined to reconstruct the image of the vincian are exposed. Vasari considers that the main characteristic is to be an artist, while Jaspers proposes that, in addition to an artist, he can be a philosopher, but by no means a scientist. From this a question arises: is it possible that Leonardo gets a place in the science, according with the Jaspers thought? And also if his verdict could be questioned. And from this, a second goal: from what science notion can our Renaissance man be considered? To answer these questions we will base on Kuhn's writings. Perhaps from a kuhnian perspective we will be able to have another vision different from the current one that considers Leonardo and his relation with the science.

Keywords: epistemology, Leonardo da Vinci, history of science. 


\section{Introducción}

Leonardo da Vinci, peculiar personaje del Renacimiento, reconocido, principalmente, por sus obras pictóricas, su afán por el conocimiento sobre la naturaleza, sus dibujos anatómicos, sus inventos, sus escritos crípticos lleno de dudas, de ansias por saber, de observaciones minuciosas. Todas estas obras del vinciano nos invitan a pensar sobre el rol científico de Leonardo. Estos múltiples intereses del renacentista mencionado pueden servir de punto disparador para pensar qué tipo de interés científico podría llegar a tener, y cuál sería su influencia y lugar dentro de la historia de la ciencia. No se pretende establecer que Leonardo sea un precursor dentro del campo científico, en todo caso se permite considerar la siguiente cuestión: ¿por qué resulta problemático introducir esta figura dentro de la historia de la ciencia? Hasta el día de hoy, se han escrito numerosos textos acerca del vinciano. Muchas interpretaciones bien argumentadas son contradictorias entre sí. En lo que respecta al interés de este trabajo, hay algunas que sostienen, por ejemplo, que Leonardo es un precursor de la ciencia moderna, otros en cambio desaprueban esta postura. Divergencias que aún no han llegado a una interpretación compartida o más aceptada. Un genio universal cuyo lugar dentro de la ciencia es discutido.

Si bien estas inquietudes son lo bastante generales y pueden ser tratas desde diferentes enfoques, en primer lugar creemos que es necesario introducir a nuestro personaje en la escenario renacentista para abordar adecuadamente los dos objetivos propuestos. El primero consistirá en una lectura entre Vasari y Jaspers observando cuáles son los aspectos que se tienen en cuenta a la hora de reconstruir una imagen del vinciano y en qué campo es ubicado por estos autores. Con respecto al primer autor, analizaremos la figura multifacética de Leonardo según la obra Le vite de' più eccellenti pittori, scultori e architettori con la intensión de ver un posible indicio de la mitificación del vinciano como genio universal y, acerca del segundo autor, revisaremos sus argumentos principales. Así, surge a modo de interrogante si es posible otorgarle un lugar dentro de la ciencia a Leonardo según la concepción de Jaspers y si su veredicto pueda entonces llegar a ser cuestionado.

Aquí se introduce el segundo objetivo, que consiste en intentar establecer en qué medida o bajo qué lectura se puede incluir a nuestro personaje en la historia de la ciencia. Para elucidar este punto se tendrán en cuenta las lecturas del capítulo 4 correspondiente a La revolución copernicana y La estructura de las revoluciones científicas de Thomas Kuhn. Quizás de este modo se obtiene una perspectiva totalmente diferente que ha hecho la tradición para el análisis de Leonardo.

\section{Escenario renacentista}

Veamos el contexto en el cual vivió Leonardo. Para tal reconstrucción nos basaremos en la Historia de la Filosofía. El pensamiento en la Edad Media y el Renacimiento de E. Paolo Lamanna y en Matemáticas para los estudiantes de humanidades de Kline Morris, introduciendo algunas frases de Leonardo según la compilación realizada por José Emilio Burucúa y Nicolás Kwiatkowski en Cuadernos de arte, literatura y ciencia. De este modo creemos poder echar un poco de luz a la ciencia vinciana.

El historiador Paolo Lamanna considera tres factores que han sido utilizados en el Renacimiento para conocer los fenómenos de la naturaleza (LAMANNA, 1960, p. 237). Uno de ellos corresponde a la filosofía, cuyos conceptos de organicidad y unidad del mundo, la distinción entre microcosmos y macrocosmos, están ligados a su vez con estudios acerca de la magia y la astrología. El segundo respecto a los inventos y descubrimientos realizados gracias a un crecimiento económico y el aumento del 
comercio entre varios países. Y por último, la nueva teoría astronómica que gestándose en Copérnico hace surgir nuevas problemáticas dentro de la cosmología.

La investigación de la naturaleza, nos comenta Lamanna, no sólo fue enriquecida por las doctrinas filosóficas fuertes adquiridas durante el Renacimiento sino también por el contexto socio-económico y político particular de la cultura de Italia, la explotación tanto de la industria como de los recursos y energías provenientes de la naturaleza en paralelo con el gran crecimiento demográfico y la necesidad de obtener medios de comunicación que fuesen más eficaces y rápidos (Ibidem, p. 240). De ello que las influyentes y poderosas familias usaran su dinero para alentar tanto a artistas como a grandes pensadores en engrandecer sus Estados ya sea por motivos de esplendor artístico, construcción de nuevas máquinas que faciliten la adquisición de productos naturales y para fines bélicos:

Todos estos factores imponían cuestiones siempre nuevas, cuestiones insolubles para el abstractismo de la ciencia escolástica dominante en las Universidades y sugerían nuevas investigaciones, experiencias y cálculos (Ibidem).

Por ello, no resulta extraño que Leonardo en una carta (DA VINCI, 2011, p. 421-424) enviada, en el año 1482, a Ludovico el Moro ofreciendo sus servicios, detalle sus estudios, proyectos e inventos en torno a este contexto mencionado, enumerando: fabricación y diseño de puentes, instrumentos para obtener agua perforando la tierra, modos de destruir fortalezas; facilidades en ataques que sean económicos, instrumentos para atacar barcos; realización de túneles ocultos; diseño de carros blindados, armas útiles pero con una estética agradable y bella y fabricación de variadas armas de guerra. Y en tiempos de paz, Leonardo explicita que puede dedicarse a la pintura, a la arquitectura y a la escultura. A partir de la lectura de esta carta podemos observar que Leonardo estaba interesado y preparado en los asuntos bélicos, y destaca también su estudio en ellos - ya sea teórico o de índole práctico según la realización de experimentos -, y apenas hace mención de su labor como artista, distante de la fama de pintor adquirida a lo largo de la historia.

Otra característica del Renacimiento, señalada por Lamanna, es la fuerte y estrecha relación entra la ciencia y el arte. El artista contratado se ocupaba tanto del diseño edificios, de las decoraciones con pinturas o figuras en mármol como de la construcción de puentes, fortalezas, etc.: "[...] de él no se exige únicamente la fantasía del artista sino la técnica del ingeniero y del mecánico [...]" (LAMANNA, 1960, p. 240). De esta exigencia surge la preparación técnica en el dibujo y el principio de la imitación de la naturaleza en las obras artísticas. Por ello el artista debe poseer conocimientos en perspectiva, matemática, geometría y óptica, además de saber acerca de los organismos de las plantas y de los animales, así como también sobre la anatomía humana. Todos estos estudios, siguiendo el análisis de Lamanna, son imprescindibles para el trabajo del artista renacentista.

Consideremos la siguiente afirmación del vinciano: "La Perspectiva es rienda y timón de la Pintura" (DA VINCI, 2011, p. 32). Asumiendo que la perspectiva es principalmente parte de la matemática y se ocupa de principios verdaderos como guía de la pintura, cuestión que afirma Leonardo diciendo que los principios verdaderos y científicos establecen las definiciones acerca de qué es una forma, cómo se compone, cómo componer un cuerpo alejado según la iluminación y las sombras, los colores, demás figuras, si están en reposo o en movimiento, etc. Dichos principios permitirían que la práctica de la Pintura sea eficaz:

Siempre la práctica debe ser edificada sobre la buena teoría, de la que la Perspectiva es guía y puerta, y sin ella nada se hace bien en los casos de la Pintura [...] (Ibidem, p. 33). 
Leonardo fue conocedor y estudioso de la perspectiva, estableciendo cuatro tipos: perspectiva lineal, perspectiva de color, perspectiva del punto de fuga y perspectiva aérea.

Sobre la relación entre el estudio de la perspectiva y la pintura, Leonardo escribe el Proemio de la perspectiva, esto es, del oficio del ojo:

[...] que con tanta claridad posee experiencia de su oficio, ha sido definido hasta mis tiempos por infinitos autores de una manera completamente diferente a la que yo he llegado gracias a la misma experiencia [...] (Ibidem, p. 103).

Aquí tenemos un indicio que además de poseer conocimientos sobre la perspectiva se debe tener conocimiento acerca de las leyes de la óptica para que el pintor en vez de errar pueda hacer una representación, en un dibujo o pintura, de la realidad lo más fiel posible a la observación de ella.

Para concluir este tópico veamos el análisis propuesto por Kline Morris acerca de la pintura relacionada con los conocimientos matemáticos y geométricos durante el Renacimiento. El autor determina que las obras artísticas producidas durante este período podrían estar exhibidas tanto en los museos de arte por en los de la ciencia. Tras los esfuerzo por los artistas de obtener un fiel realismo de la naturaleza se dedicaron a la descripción y a la imitación lo más acorde posible en sus obras. El objetivo es, y tal como lo expresa Leonardo, la reproducción fiel y exacta de la naturaleza. Para tal objetivo, los renacentistas adquirieron el ideal griego de que "en las matemáticas estaba la esencia del mundo real" (KLINE, 2012, p. 253), y por ello debieron adquirir leyes matemáticas que permitan plasmar en una obra artística la realidad observada.

Kline Morris determina que muchos artistas contribuyeron a la creación de un sistema matemático de perspectiva, iniciándose con Brunelleschi y perfeccionándose con Uccello, della Francesca, da Vinci, Durero, entre otros. Ellos idearon tantas técnicas para que el pintor en una obra pueda realizar proyección de las líneas de la perspectiva del objeto al lienzo como teoremas matemáticos sobre el dibujo en perspectiva para lograr las escenas lo más realista posibles:

En la historia de la cultura las realizaciones de los artistas del Renacimiento tienen gran significación. Su propósito manifiesto fue el de contemplar la naturaleza y describir sobre el lienzo lo que veían, pero su verdadero e íntimo propósito fue el de descubrir los secretos mismos de la naturaleza. El artista del Renacimiento fue hombre de ciencia, y pintar fue ciencia no tan sólo por su contenido sumamente técnico y aun matemático, sino también porque se inspiraba en la finalidad primordial de la ciencia: la comprensión de la naturaleza (Ibidem, p. 267).

Dentro del amplio interés de Leonardo sobre el mundo natural, vemos que incursionó por los campos de la mecánica, la matemática, la física, la fisionomía, la astronomía, la anatomía, entre otros. Lamanna comenta que en los escritos del vinciano en los cuales registraba sus observaciones y estudios

[...] se anticipan, o por lo menos se presienten, muchas verdades e invenciones que solamente en siglos sucesivos debían tener el reconocimiento o la consagración definitiva [...] (LAMANNA, 1960, p. 243).

Leonardo nos dice que si queremos certidumbre alguna debemos ir a la experiencia, si elaboramos reglas generales debemos probarlas en la experiencia varias veces con el objetivo de saber si dichas reglas se corresponden a tal realidad observada. Para el vinciano es menester estudiar primero la ciencia porque sólo obtendremos certidumbre si logramos aplicar las ciencias matemáticas. Por esto, no resulta extraño que Leonardo sostenga que ningún tipo de investigación de índole científico debe considerarse parte de la ciencia verdadera si no posee demostraciones matemáticas y que estas deban ser aplicadas a la experiencia puesto que "[...] la experiencia no falla 
jamás, solo fallan nuestros juicios cuando esperan de ella efectos tales que nuestros experimentos no causan [...]" (DA VINCI, 2011, p. 156), agregando en otro pasaje que "quien espera de la experiencia lo que no está en ella, se aparta de la razón" (Ibidem). Para el vinciano se debe partir de la experiencia y luego mediante la razón demostrar las causas o leyes de tal experiencia. De esta concepción se parte el rol primordial de la experiencia que para Leonardo es aquella que hace de mediadora entre la naturaleza y los seres humanos.

\section{El origen del mito: un artista considerado genio universal}

En 1550 es publicada Le vite de' più eccellenti pittori, scultori e architettori. Con más de novecientas páginas, según la versión digital disponible, no es sólo un texto que abarque una recolección de biografías de artistas italianos de antaño. En su carácter de historiador Vasari proporciona información técnica y precisa sobre el arte en la época renacentista italiana. Ya en el Proemio hace mención de tres disciplinas artísticas primordiales, a saber: pintura, escultura y arquitectura, además de abordar una discusión de aquel entonces que consistía en cuáles de éstas sería la más importante o la más completa, y qué relaciones se establecen entre ellas. Luego, se encuentran capítulos dedicados especialmente a estas tres disciplinas artísticas, cuyo tratamiento de estas es bastante extenso, y resultan esclarecedores para ver en qué consistían la arquitectura, pintura, escultura en la Italia renacentista. Por último, Vasari analiza las relaciones existentes entre los distintos artistas estudiados, el medio en el cual se establecen los vínculos entre los artistas con sus respectivos maestros.

Además los artistas expuestos son agrupados en tres partes cronológicas establecidas por el autor. Entre los artistas mencionados por Vasari se encuentra Leonardo da Vinci, a quién a cuya vida y obra le dedicó aproximadamente catorce páginas. Creemos que el mito sobre Leonardo se basa en pocas páginas suponiendo que su origen se puede rastrear en dicha obra. El autor comenta que hay ciertos rasgos novedosos o mejorados con respecto a la pintura en el vinciano.

La descripción proporcionada por Vasari versa sobre la labor artística de Leonardo en la pintura. No encontramos mención o cita alguna sobre los escritos propios de Leonardo o acerca de sus diseños, como por ejemplo, las máquinas y otras cuestiones que no sean referentes principalmente a la pintura. Tampoco observamos fechas aproximadas de su nacimiento, muerte, viajes, etc. Ni referencia a otras relaciones que tuvo Leonardo con sus contemporáneos o su interés por cuestiones militares. No obstante, se destaca su carácter sociable, el cual le permitía mantener vínculos con personas de mucha influencia en la época, por ejemplo: Familia Brunelleschi, i Medici, Ludovico il Moro, Cesare Borgia, entre otros.

Siguiendo la lectura vemos a Leonardo como una persona que tuvo talento para las artes desde joven, en el taller de Verrocchio aprendió las artes de su maestro a quien admiró y en muchos aspectos llegó a superar. Se destacó por la perfección de sus obras en las que se pueden enfatizar el carácter divino de los trazos y figuras de nuestro artista. En palabras del historiador italiano:

Grandissimi doni si veggono piovere da gli influssi celesti ne' corpi umani molte volte naturalmente; e sopra naturali tavolta strabocchevolmente accozarsi in un copor solo belleza, grazia e vitrtú, in una maniera che dovunque si volge quel tale [...] E per esperienzia si vede quegli che con qualche studio accidentale si volgono a seguire l'orme di questi mirabili spiriti, se punto sono dalla natura aiutati, quando il medesimo non sono che essi, tanto almanco s'accostano a le divine opere loro. Che participano di quella divinità (VASARI, 1986, p. 554). 
De este modo, el vinciano es presentado como un hombre talentoso, ingenioso y virtuoso pero principalmente como artista. Sin embargo, Leonardo tuvo otros intereses y demostró grandes habilidades para ello, tanto en la arquitectura, la música, la geometría, el estudio de la naturaleza y del cielo. Vasari describe ciertos rasgos de su personalidad, particularmente de ser una persona alegre, sociable, generosa aunque con respecto a la producción de sus obras era visto como alguien caprichoso. Quizá pudo ser considerado caprichoso por este múltiple interés en otras disciplinas que no fuesen la pintura hacía que dejara de lado sus trabajos artísticos inconclusos, también por el motivo de que no se contentaba fácilmente con sus propias creaciones, dejando entrever que Leonardo en algunas oportunidades desaprovechó su talento artístico. No obstante, Vasari menciona algunos de sus cuadros de renombre, una descripción técnica de ellos y la recepción que tuvieron por parte del público y del entorno en el cual Leonardo se encontraba.

La conclusión a la que llega el historiador italiano acerca de las pinturas de Leonardo pueden resumirse diciendo que lo considera como un genio universal y que el carácter de su arte es más divino que humano, como se puede observar en la cita anteriormente mencionada. De esto, agrega que en sus pinturas se puede ver cuánto el arte puede imitar a la naturaleza. Por esta excelencia de sus obras, la fama de Leonardo creció, destacándose también por sus dibujos en anatomía. Gracias a Vasari disponemos de algunas anécdotas pintorescas, una de ellas cuenta que Leonardo mientras caminaba por la calle realizaba figuras de animales con un tipo de cera liviana y los hacía volar por el aire. Podemos ver que es notable la admiración hacia Leonardo por parte de este historiador italiano. Y si bien puede ser que su figura sea engrandecida o en ciertos aspectos divinizada por este, no hay que dejar de lado que el historiador de arte italiano además de realizar una contemplación estética de Leonardo también destaca los aspectos técnicos en la pintura.

\section{Un filósofo apartado de la ciencia moderna}

Discutir sobre la figura de Leonardo como artista entendemos que carece prácticamente de sentido. Ahora bien, ¿hay otro Leonardo? Un Leonardo que además de ser artista es también un filósofo e inventor. Una imagen más unificada del vinciano es aquella que propone Karl Jaspers en su obra Leonardo como filósofo. Tras la lectura de la obra dos cuestiones fundamentales que merecen su debida atención: la primera abarca las diferencias y similitudes de Leonardo con respecto a la ciencia moderna y la segunda consiste en analizar la interpretación que realiza Jaspers al respecto. Para ello se debe tener presente que debemos considerarlo a la vez pintor, inventor y filósofo.

Comencemos por la primera cuestión señalada: qué rasgos o contenidos dentro del pensamiento de Leonardo se pueden o no considerar como caracteres principales referentes a la ciencia, en particular, la ciencia moderna. Para responder este punto, Jaspers propone indicar en qué consistía el conocimiento según la perspectiva vinciana. El autor reconstruye de la siguiente manera el modo de conocer de nuestro personaje: todo conocer, la actividad cognoscitiva, está referida al ojo y a la mano: "[...] lo que existe debe ser visible y lo que se conoce tiene que ser reproducido con las manos [...]" (JASPERS, 1956, p. 15). Entonces, para tal conocimiento sobre lo sensible y su correspondiente percepción y reproducción, según la lectura de Jaspers sobre Leonardo, es necesario tener una base matemática a través, quizás, del arte. Sobre ello el autor comenta que tanto la mecánica como la matemática para el vinciano pertenecen a

[...] un mundo de lo visible que puede ser reproducido con las manos en el espacio y sobre el cual es posible influir directamente o por medio de máquinas fabricadas por el hombre [...] (Ibidem, p. 37), 
es decir, se trata de captar la espiritualidad de lo sensible mediante la vista y reproducirlo con la mano haciendo de la observación de los fenómenos el hilo conductor del conocimiento.

Pero antes de continuar cabe preguntarse ¿qué entiende Jaspers por ciencia moderna? El autor proporciona algunos tópicos: el conocimiento mediante reproducción basándose en figuras morfológicas en el caso de la anatomía, geología y botánica. A su vez, el conocimiento se basa, también, en la percepción sensible, que no es la mera contemplación de los fenómenos de la experiencia. Además, se busca que el conocimiento sea cierto y válido característico de un investigador moderno. La ciencia moderna, por ende, pretende ser universal y progresiva, esta visión de la ciencia es la que adopta Jaspers al realizar su veredicto (Ibidem, p. 31-32).

Otra cuestión que destacamos, bajo la lectura jaspersiana, advierte que los descubrimientos de Leonardo no tienen validez según la perspectiva moderna porque aquellos "[...] son productos de la contemplación óptica de las cosas, bajo la influencia de una conciencia del mundo que lo abarca todo [...]" (Ibidem, p. 33) y que no pueden comprenderse de un modo metódico y sistemático. Del mismo modo, el autor duda si el vinciano realmente pudo comprender en que consistía la matemática, alegando que para nuestro personaje renacentista solamente significó una imagen intuitiva que se expresa mediante formas geométricas. Es así que al no disponer de un método que dirija sus investigaciones u observaciones y al no poseer una teoría que sustente aquellas, parecería que a Leonardo le estaría vedado el ingreso al mundo científico. No obstante, el autor considera que:

Tomar a Leonardo como espíritu de la ciencia moderna, es acertado; tomarlo como símbolo de las ciencias naturales con criterio matemático resulta erróneo. Esta circunstancia nos recuerda que la matemática de la naturaleza es tan sólo un momento y no la medida verdadera de la enorme construcción de la ciencia actual (Ibidem, p. 38).

Siguiendo el veredicto de Jaspers, surge el interrogante si acaso se lo debe pensar a Leonardo no únicamente como ejemplo del espíritu de la ciencia moderna, sino también como científico de su época, es decir, renacentista. Además, si el propio autor está hablando de una ciencia característica o propia de Leonardo, no queda claro entonces respecto en qué tipo de ciencia se lo incluye o no. En todo caso, ¿considerar al vinciano científico o no, depende solamente de una definición de ciencia? En nuestra opinión no sólo radica en una diferencia entre definiciones. Habrá que analizar algo más lo cual será tratado en el siguiente apartado.

\section{¿Leonardo científico?}

Retomando estas últimas preocupaciones en las cuales Leonardo ya no es visto sólo como un artista sino también como un filósofo, surgen los siguientes interrogantes: ¿al no disponer un método científico, debemos excluir del mundo de las ciencias? ¿O Jaspers estará en este punto influenciado por la tradición? En todo caso, el vinciano debería pensarse quizá en su propio contexto histórico, estableciendo que aspectos tiene de la época pasada y cuáles posteriores a la suya. Así también surge el interrogante siguiente: ¿Se lo podría considerar un científico, sin entrar por ahora en cuestiones de definiciones, renacentista?

En La estructura de las revoluciones científicas Thomas Kuhn realiza la siguiente pregunta: “ ¿Por qué la empresa bosquejada antes habría de avanzar uniformemente de una manera que le está vedada digamos al arte, la teoría política o la filosofía?" (KUHN, 2007, p. 281). Esta cuestión nos indica qué tan difíciles y complicadas pueden llegar a ser las conexiones entre las nociones de progreso y ciencia. Acerca del renacimiento, el autor comenta que la pintura era una disciplina acumulativa, 
los artistas tenían como fin la representación por ello, los historiadores de la época se centraban en registrar y detallar cada avance o técnica que mejoraba o posibilitaba una representación más adecuada de la naturaleza. A su vez, en dicha época no había una separación tajante entre disciplinas artísticas o científicas, sobre Leonardo comenta que era un persona más de las que "[...] pasaban libremente de un campo a otro, campos que sólo más tarde se convertirían en terrenos categóricamente distintos [...]" (Ibidem, p. 283).

Por cuestiones de espacio e interés nos centraremos en algunos puntos tratados por Kuhn. En unos de los últimos capítulos de La estructura de las revoluciones científicas se plantea la cuestión acerca de las revoluciones científicas invisibles. Muchas veces, comenta el autor, invisibilidad es debido a la imagen de la ciencia o de la actividad científica como fuente autorizada. Kuhn se refiere que esta fuente de autoridad se encuentra principalmente a los textos de divulgación científica y de filosofía para ocultar ciertas revoluciones, por ejemplo en aquellos libros de textos que usualmente son escritos para los estudiantes de una determinada disciplina (Ibidem, p. 247) ${ }^{1}$.

Dichos textos vemos que están escritos según los criterios la comunidad científica de un momento determinado, lo cual permite observar que tanto los problemas, teorías, conceptos tratados y establecidos serán aquellos que son aceptados por dicha comunidad. En dichos textos, que por un lado son útiles como herramientas pedagógicas, el vocabulario empleado en los libros de textos será el lenguaje científico contemporáneo correspondiente a la comunidad científica, olvidándose el significado en un sentido histórico de los conceptos utilizados en épocas pasadas. Del mismo modo nos encontramos con las divulgaciones de índole científicas que pretender utilizar un vocabulario más cotidiano, ameno para comunicar aquellos contenidos científicos. Y en el caso de los textos correspondientes a la filosofía, procuran un análisis de la estructura lógica de aquellos conocimientos considerados científicos. Para Kuhn estos tipos de textos "[...] registran el resultado estable de las revoluciones pretéritas, mostrando de ese modo las bases de la tradición de la ciencia normal vigente [...]" (Ibidem, p. 248). Al ser presentadas las figuras del pasado como aquellas que contribuyeron al desarrollo de la ciencia o de una disciplina en particular vigente, se ve que los conjuntos ya sea de teorías, de conceptos, o de las problemáticas planteadas tienen un carácter fijo.

Creemos que podemos ubicar a Jaspers en una interpretación de este tipo. Hemos visto que este autor no considera a Leonardo un científico por no poseer método, sistema o rigurosidad exigida por la ciencia. También observamos que Jaspers traslada una concepción establecida y tradicional de la ciencia y de la actividad científica a un momento pretérito como lo es el Renacimiento. Al respecto Kuhn sostiene que

[...] los resultados de la investigación científica no muestran una dependencia obvia del contexto histórico de la indagación y en parte porque, excepto durante las crisis y las revoluciones, la posición contemporánea de los científicos parece ser muy segura [...] (Ibidem, p. 250).

Esta tendencia que nos marca Kuhn no se refiere tanto al hecho de que al hacer historia de la ciencia, por ejemplo, haya un olvido de los héroes pasados sino que hay un olvido o una falta de revisión de las obras de dichos héroes, pretendiendo que la reconstrucción de dicha historia sea vista de un modo lineal y acumulativo. Con ello, queremos hacer notar, como sostiene Kuhn, el modo en que se desarrollan la ciencia o la construcción de una historia de la ciencia no es acumulativo pues los cánones presentes no corresponden a los problemas y nociones pasadas: "Las generaciones anteriores se plantearon sus propios problemas con sus propios instrumentos y sus propios cánones de solución [...]" (Ibidem, p. 254).

\footnotetext{
${ }^{1}$ Acerca de los libros académicos consultamos particularmente el capítulo XI de La estructura...: La invisibilidad de la revoluciones. Ibidem, p. 247 y ss.
} 
Tengamos esto último mencionado para considerar la siguiente situación. En $L a$ revolución copernicana, en el capítulo cuatro, Kuhn nos invita a pensar qué sucede si consideramos a Copérnico como heredero directo de Ptolomeo y Aristóteles. $\mathrm{Si}$ hacemos este ejercicio que nos propone nos va a parecer que durante trece siglos no hubo actividad científica o legado que sea de interés para el desarrollo de la teoría copernicana. Sin embargo, el autor nos indica que estos trece años fueron intermitentes. A lo largo de aquellos siglos, se recuperaron las obras de los antiguos griegos, se discutieron, se tradujeron en varias lenguas, se interpretaron, y asíllegaron a las manos de Copérnico, esto quiere decir que los autores griegos no se congelaron a lo largo del tiempo para llegar intactos. En ese ínterin demasiado amplio hubo nociones o interpretaciones que ayudaron a Copérnico a establecer su nuevo sistema astronómico:

[...] la actividad científica, aunque intermitente, fue muy intensa durante esta época y desempeñó un papel esencial en la preparación del terreno para el nacimiento y posterior triunfo de la revolución copernicana [...] (KUHN, 1978, p. 143).

De esta exposición kuhniana mencionaremos el ejemplo del Sol. Nuestra hipótesis es la siguiente: Leonardo formó parte del debate acerca del culto al sol característico de su época y este culto interviene en la elaboración de un nuevo sistema astronómico que tendrá sus inicios en la teoría copernicana. Veamos con un poco de detenimiento este último punto que nos servirá quizás a echar luz a la ciencia vinciana dentro de la historia de la ciencia al modo kuhniano que esbozamos.

Aquello que plantea Kuhn sobre el sol es acerca de la vinculación entre el neoplatonismo y el culto al sol, aquí hace referencia a Proclo y su concepción del dios neoplatónico representada su divinidad materialmente en el sol. Kuhn observa que tanto en el arte como en literatura del renacimiento encontramos una identificación simbólica de Dios con el sol, tomando por ejemplo unos fragmentos de una obra de Ficino, contemporáneo de Leonardo, donde se establece una relación simbólica sol-Dios: “El sol puede significar para vosotros el propio Dios, ¿y quién osaría decir que el sol es una simple ilusión?" (FICINO, 1978, p. 180), siendo éste que posee una luz que deja ver la naturaleza de aquel ya que la luz es aquella que se difunde más rápidamente y pasa por todas las cosas sin causar daño, mostrando el amor de dios; su calor como fuente de crecimiento de la naturaleza. Si bien este culto al sol está alejado de la ciencia Kuhn ve un punto de contacto entre el neoplatonismo renacentista y el nuevo sistema astronómico encaminado por Copérnico:

No obstante, la susodicha ubicación no era compatible con el sistema astronómico de Ptolomeo, y las dificultades planteadas al neoplatonismo por tal desacuerdo quizá ayudaran a Copérnico en su concepto de un nuevo sistema edificado alrededor de un sol central. [...] dichas dificultades le proporcionaron un argumento en favor de su nuevo sistema [...] (Ibidem).

Ahora nos resta ver como Leonardo está inserto en esa problemática que no era ajeno al pensamiento renacentista. Tenemos que buscar indicios o elementos significativos para una revolución ampliando contexto intelectual, analizar las opiniones, las discusiones, las problemáticas de dicho contexto. Por ello, iremos de nuevo directamente a las fuentes del vinciano. Leonardo esboza las propiedades del sol, estudios sobre su tamaño, sobre la luz de planetas y estrellas nacidos de la propia luz del sol, algunos de sus estudios se basan en lecturas de algunos fragmentos extraídos de Epicuro. Y también realiza un elogio, similar al de Ficino:

[...] su luz ilumina a todos los cuerpos celestes que recorren el universo, todas las amas descienden de él, porque el calor en los animales vivos viene de las almas, y ningún otro calor ni luz hay en el universo [...] (DA VINCI, 2011, p. 170-171). 
De hecho al final de su fragmento el vinciano hace alusión que muchos otros honran al sol. De hecho, en estos "otros", tal como indican los traductores de la edición que utilizamos de la obra vinciana, se podría estar aludiendo a Ficino y demás pensadores pertenecientes al núcleo platónico o neoplatónico (Ibidem, p. 171)2. Hay una frase que, siguiendo el análisis kuhniano, sirve para demostrar que el pensamiento del vinciano no era compatible con el sistema ptolemaico: "El sol no se mueve" (Ibidem), una breve sentencia de Leonardo. De este modo, creemos que con un futuro análisis minucioso de los escritos de Leonardo podremos, quizás, sostener nuestra hipótesis y afirmar que nuestro personaje renacentista aportó de alguna manera a la transición de dichos sistemas astronómicos y que el vinciano forme parte. En palabras de Kuhn:

En el ínterin, el propio proceso de descubrimiento de la misma, la integración medieval de la ciencia y la teología, los siglos de crítica escolástica y las nuevas corrientes de pensamiento y formas sociales surgidas en el Renacimiento se combinaron para cambiar la actitud de los hombres de su época frente a la herencia científica que aprendían en las universidades (Ibidem, p. 189).

De este modo, podemos concluir que Jaspers tenía una concepción de la ciencia de su momento, basada en la teoría y principios matemáticos principalmente, y que esta concepción fuese pretendida en Leonardo. Sin embargo, afirmamos que el vinciano se interesa por la matemática pero más por la aplicación de ella a objetos materiales, ya sea, por ejemplo, aplicando la perspectiva en la pintura o aplicando la mecánica en algunos de sus inventos. Y junto a la lectura de Kuhn vimos que hay cuestiones y problemáticas que deben analizarse desde las concepciones del momento dado, por ello creemos que el veredicto de Jaspers puede ser cuestionado y discutido. Además sugerimos que el problema surge, en parte, por el tipo de comunicación entre los científicos y los textos de divulgación. Entonces si pretendemos colocar a Leonardo dentro de las discusiones referentes a la ciencia debemos ir al contexto intelectual de la época, revisar tanto las obras de él como las de sus contemporáneos, ver qué se entendía por este o aquel concepto, fenómeno, etc., cuáles eran las problemáticas y las soluciones que se daban a aquellas, qué discusiones había, en qué debates nuestro renacentista estaba inserto. Expusimos el ejemplo del sol para demostrar que el vinciano no era ajeno a las discusiones de su época, que tenía una propuesta propia para dar, y buscamos cierta huella vinciana que haya aportado o ayudado a la creación del terreno para lo que luego sería la revolución copernicana.

Correspondencia: Maíra Pouey Bragos. Universidad Nacional de Rosario, Maipu 1065, 2000, Rosario, Santa Fe, Argentina. E-mail: mpoueybragos@gmail.com

Conflicto de intereses: Ninguno.

Todos los autores han leído y aprobado la versión final remitida a la revista Em curso.

\footnotetext{
${ }^{2}$ Ver nota a pie de pág. n²1. Ibidem, p. 171.
} 


\section{Bibliografía}

DA VINCI, L. Cuadernos de arte, literatura y ciencia. Traducción de José Emilio Burucúa y Nicolás Kwiatkowski. Buenos Aires: Colihue, 2011. v. 2.

FICINO, M. Liber de sole. In: KUHN, T. La revolución copernicana. Madrid: Orbis, 1978. p. 180

JASPERS, K. Leonardo como filósofo. Traducción de Jorge Oscar Pickenhayn. Buenos Aires: Sur, 1956.

KLINE, M. Matemáticas para los estudiantes de humanidades. Traducción de Roberto Elier y Raúl Zamora. México: Fondo de Cultura Económica, 2012.

KUHN, T. La revolución copernicana. Traducción de Domenc Bergada. Madrid: Orbis, 1978. v. 1.

KUHN, T. La estructura de las revoluciones científicas. Traducción de Carlos Solís Santos. México: Fondo de Cultura Económica, 2007.

LAMANNA, P. Historia de la filosofía: el pensamiento en la Edad Media y el Renacimiento. Traducción de Oberdan Caletti. Buenos Aires: Hachette, 1960.

VASARI, G. Le vite de' più eccellenti pittori, scultori e architettori. Torino, 1986. Letteratura italiana Einaud. Disponible en http: www.letteraturaitaliana.net/pdf/Volume_5/t129.pdf. Acceso en: julio de 2015. 